\title{
A Construction of Small $(q-1)$-Regular Graphs of Girth 8 *
}

\author{
M. Abreu ${ }^{\dagger}$ \\ Dipartimento di Matematica, \\ Informatica ed Economia \\ Università degli Studi della Basilicata \\ I-85100 Potenza, Italy \\ marien.abreu@unibas.it \\ C. Balbuena ${ }^{\S}$ \\ Departament de Matemática Aplicada III \\ Universitat Politècnica de Catalunya \\ E-08034 Barcelona, Spain \\ m. camino. balbuena@upc. edu
}

\author{
G. Araujo-Pardo $\ddagger$ \\ Instituto de Matemáticas \\ Campus Juriquilla \\ Universidad Nacional Autónoma de México \\ Juriquilla 76230, Querétaro, México \\ garaujo@matem.unam.mx \\ D. Labbate ${ }^{\dagger}$ \\ Dipartimento di Matematica, \\ Informatica ed Economia \\ Università degli Studi della Basilicata \\ I-85100 Potenza, Italy \\ domenico.labbate@unibas.it
}

Submitted: May 24, 2014; Accepted: Apr 6, 2015; Published: Apr 21, 2015

Mathematics Subject Classifications: 05C35, 05C69

\begin{abstract}
In this note we construct a new infinite family of $(q-1)$-regular graphs of girth 8 and order $2 q(q-1)^{2}$ for all prime powers $q \geqslant 16$, which are the smallest known so far whenever $q-1$ is not a prime power or a prime power plus one itself.
\end{abstract}

Keywords: Cages, girth, Moore graphs, perfect dominating sets

\section{Introduction}

Throughout this note, only undirected simple graphs without loops or multiple edges are considered. Unless otherwise stated, we follow the book by Bondy and Murty [11] for terminology and notation.

\footnotetext{
${ }^{*}$ Research supported by CONACyT-México under project 178395.

${ }^{\dagger}$ Research supported by the Italian Ministry MIUR and carried out within the activity of INdAM-GNSAGA.

${ }^{\ddagger}$ Research supported by CONACyT-México under projects 178395, 166306, and PAPIIT-México under project IN104915.

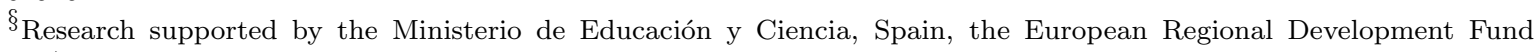
(ERDF) under project MTM2011-28800-C02-02; and under the Catalonian Government project 1298 SGR2009.
} 
Let $G$ be a graph with vertex set $V=V(G)$ and edge set $E=E(G)$. The girth of a graph $G$ is the number $g=g(G)$ of edges in a smallest cycle. For every $v \in V$, $N_{G}(v)$ denotes the neighbourhood of $v$, that is, the set of all vertices adjacent to $v$. The degree of a vertex $v \in V$ is the cardinality of $N_{G}(v)$. Let $A \subset V(G)$, we denote by $N_{G}(A)=\cup_{a \in A} N_{G-A}(a)$ and by $N_{G}[A]=A \cup N_{G}(A)$. For $v, w \in V(G)$ denote by $d(v, w)$ the distance between $v$ and $w$. Moreover, denote by $N^{m}(v)=\{w \in V(G) \mid d(v, w)=m\}$ and $N^{m}[v]=\{w \in V(G) \mid d(v, w) \leqslant m\}$ the $m^{\text {th }}$ open and closed neighbourhood of $v$ respectively.

A graph is called regular if all the vertices have the same degree. A $(k, g)$-graph is a $k$-regular graph with girth $g$. Erdős and Sachs [12] proved the existence of $(k, g)$-graphs for all values of $k$ and $g$ provided that $k \geqslant 2$. Since then most work carried out has focused on constructing a smallest one (cf. e.g. [1, 2, 3, 4, 5, 6, 7, 9, 13, 15, 18, 20, 21]). A $(k, g)$-cage is a $k$-regular graph with girth $g$ having the smallest possible number of vertices. Cages have been intensively studied since they were introduced by Tutte [23] in 1947. More details about constructions of cages can be found in the survey by Exoo and Jajcay [14].

In this note we are interested in $(k, 8)$-cages. Counting the number of vertices in the distance partition with respect to an edge yields the following lower bound on the order of a $(k, 8)$-cage:

$$
n_{0}(k, 8)=2\left(1+(k-1)+(k-1)^{2}+(k-1)^{3}\right) .
$$

A $(k, 8)$-cage with $n_{0}(k, 8)$ vertices is called a Moore $(k, 8)$-graph (cf. [11]). These graphs have been constructed as the incidence graphs of generalized quadrangles of order $k-1$ (cf. [9]). All these objects are known to exist for all prime power values of $k-1$ (cf. e.g. $[8,16]$ ), and no example is known when $k-1$ is not a prime power. Since they are incidence graphs, these cages are bipartite and have diameter 4 .

A subset $U \subset V(G)$ is said to be a perfect dominating set of $G$ if for each vertex $x \in V(G) \backslash U,\left|N_{G}(x) \cap U\right|=1$ (cf. [17]). Note that if $G$ is a $(k, 8)$-graph and $U$ is a perfect dominating set of $G$, then $G-U$ is clearly a $(k-1)$-regular graph, of girth at least 8. Using classical generalized quadrangles, Beukemann and Metsch [10] proved that the cardinality of a perfect dominating set $B$ of a Moore $(q+1,8)$-graph, $q$ a prime power, is at most $|B| \leqslant 2\left(2 q^{2}+2 q\right)$ and if $q$ is even $|B| \leqslant 2\left(2 q^{2}+q+1\right)$.

For $k=q+1$ where $q \geqslant 2$ is a prime power, we find a perfect dominating set of cardinality $2\left(q^{2}+3 q+1\right)$ for all $q$ (cf. Proposition 2$)$. This result allows us to explicitly obtain $q$-regular graphs of girth 8 and order $2 q\left(q^{2}-2\right)$ for any prime power $q$ (cf. Definition 3 and Lemma 4). Finally, we prove the existence of a perfect dominating set of these $q$ regular graphs which allow us to construct a new infinite family of $(q-1)$-regular graphs of girth 8 and order $2 q(q-1)^{2}$ for all prime powers $q$ (cf. Theorem 5), which are the smallest known so far for $q \geqslant 16$ whenever $q-1$ is not a prime power or a prime power plus one itself. Previously, the smallest known $(q-1,8)$-graphs, for $q$ a prime power, were those of order $2 q\left(q^{2}-q-1\right)$ which appeared in [7]. The first ten improved values appear in the following table in which $k=q-1$ is the degree of a $(k, 8)$-graph, and the other columns contain the old and the new upper bound on its order. 


\begin{tabular}{|c|c|c|c|c|c|}
\hline$k$ & Bound in [7] & New bound & $k$ & Bound in [7] & New bound \\
\hline 15 & 7648 & 7200 & 52 & 292030 & 286624 \\
\hline 22 & 23230 & 22264 & 58 & 403678 & 396952 \\
\hline 36 & 98494 & 95904 & 63 & 515968 & 508032 \\
\hline 40 & 134398 & 131200 & 66 & 592414 & 583704 \\
\hline 46 & 203134 & 198904 & 70 & 705598 & 695800 \\
\hline
\end{tabular}

\section{Construction of small $(q-1)$-regular graphs of girth 8}

In this section we construct $(q-1)$-regular graphs of girth 8 with $2 q(q-1)^{2}$ vertices, for every prime power $q \geqslant 4$. To this purpose we need the following coordinates for a Moore $(q+1,8)$-cage $\Gamma_{q}$.

Definition 1. $[19,22]$ Let $\mathbb{F}_{q}$ be a finite field with $q \geqslant 2$ a prime power and $\varrho$ a symbol not belonging to $\mathbb{F}_{q}$. Let $\Gamma_{q}=\Gamma_{q}\left[V_{0}, V_{1}\right]$ be a bipartite graph with vertex sets $V_{i}=$ $\mathbb{F}_{q}^{3} \cup\left\{(\varrho, b, c)_{i},(\varrho, \varrho, c)_{i}: b, c \in \mathbb{F}_{q}\right\} \cup\left\{(\varrho, \varrho, \varrho)_{i}\right\}, i=0,1$, and edge set defined as follows:

For all $a \in \mathbb{F}_{q} \cup\{\varrho\}$ and for all $b, c \in \mathbb{F}_{q}$ :

$$
\begin{aligned}
& N_{\Gamma_{q}}\left((a, b, c)_{1}\right)= \begin{cases}\left\{\left(w, a w+b, a^{2} w+2 a b+c\right)_{0}: w \in \mathbb{F}_{q}\right\} \cup\left\{(\varrho, a, c)_{0}\right\} & \text { if } a \in \mathbb{F}_{q} ; \\
\left\{(c, b, w)_{0}: w \in \mathbb{F}_{q}\right\} \cup\left\{(\varrho, \varrho, c)_{0}\right\} & \text { if } a=\varrho .\end{cases} \\
& N_{\Gamma_{q}}\left((\varrho, \varrho, c)_{1}\right)=\left\{(\varrho, c, w)_{0}: w \in \mathbb{F}_{q}\right\} \cup\left\{(\varrho, \varrho, \varrho)_{0}\right\} \\
& N_{\Gamma_{q}}\left((\varrho, \varrho, \varrho)_{1}\right)=\left\{(\varrho, \varrho, w)_{0}: w \in \mathbb{F}_{q}\right\} \cup\left\{(\varrho, \varrho, \varrho)_{0}\right\} .
\end{aligned}
$$

Or equivalently

For all $i \in \mathbb{F}_{q} \cup\{\varrho\}$ and for all $j, k \in \mathbb{F}_{q}$ :

$$
\begin{aligned}
& N_{\Gamma_{q}}\left((i, j, k)_{0}\right)= \begin{cases}\left\{\left(w, j-w i, w^{2} i-2 w j+k\right)_{1}: w \in \mathbb{F}_{q}\right\} \cup\left\{(\varrho, j, i)_{1}\right\} & \text { if } i \in \mathbb{F}_{q} ; \\
\left\{(j, w, k)_{1}: w \in \mathbb{F}_{q}\right\} \cup\left\{(\varrho, \varrho, j)_{1}\right\} & \text { if } i=\varrho .\end{cases} \\
& N_{\Gamma_{q}}\left((\varrho, \varrho, k)_{0}\right)=\left\{(\varrho, w, k)_{1}: w \in \mathbb{F}_{q}\right\} \cup\left\{(\varrho, \varrho, \varrho)_{1}\right\} ; \\
& N_{\Gamma_{q}}\left((\varrho, \varrho, \varrho)_{0}\right)=\left\{(\varrho, \varrho, w)_{1}: w \in \mathbb{F}_{q}\right\} \cup\left\{(\varrho, \varrho, \varrho)_{1}\right\} .
\end{aligned}
$$

Note that $\varrho$ is just a symbol not belonging to $\mathbb{F}_{q}$ and no arithmetical operation will be performed with it. Figure 1 shows a spanning tree of $\Gamma_{q}$ with the vertices labelled according to Definition 1.

Proposition 2. Let $q \geqslant 2$ be a prime power and let $\Gamma_{q}=\Gamma_{q}\left[V_{0}, V_{1}\right]$ be the Moore $(q+1,8)$ graph with the coordinates as in Definition 1. Let $A=\left\{(\varrho, 0, c)_{1}: c \in \mathbb{F}_{q}\right\} \cup\left\{(\varrho, \varrho, 0)_{1}\right\}$ and let $x \in \mathbb{F}_{q} \backslash\{0\}$. Then the set

$$
N_{\Gamma_{q}}[A] \cup\left(\bigcap_{a \in A} N_{\Gamma_{q}}^{2}(a)\right) \cup N_{\Gamma_{q}}^{2}\left[(\varrho, \varrho, x)_{1}\right]
$$

is a perfect dominating set of $\Gamma_{q}$ of cardinality $2\left(q^{2}+3 q+1\right)$. 


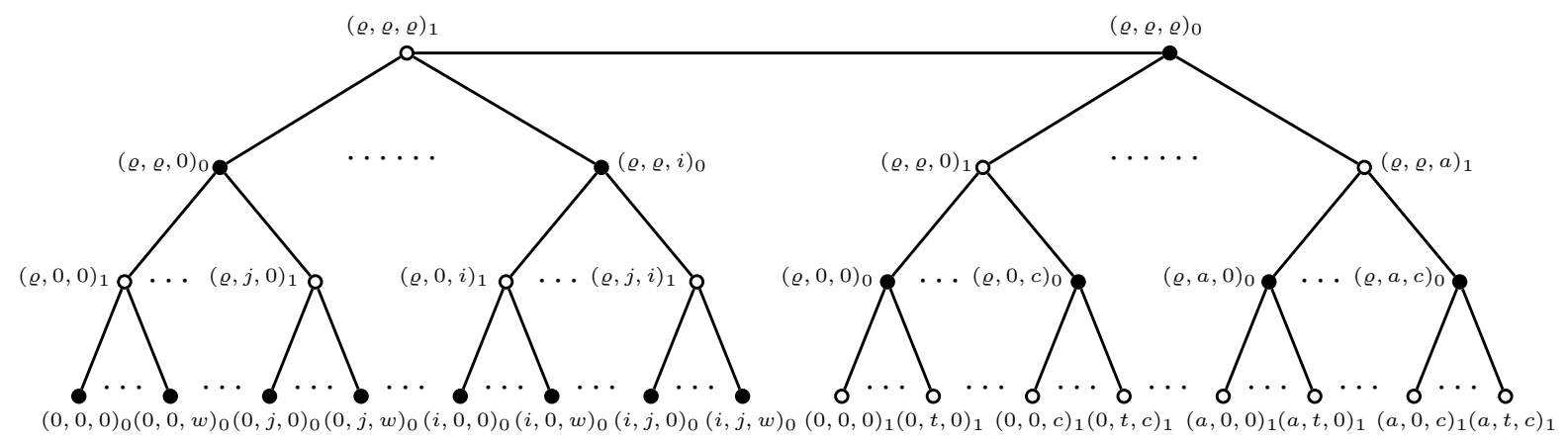

Figure 1: Spanning tree of $\Gamma_{q}$.

Proof. From Definition 1, it follows that $A=\left\{(\varrho, 0, c)_{1}: c \in \mathbb{F}_{q}\right\} \cup\left\{(\varrho, \varrho, 0)_{1}\right\}$ has cardinality $q+1$ and its elements are mutually at distance four. Then $\left|N_{\Gamma_{q}}[A]\right|=(q+$ $1)^{2}+q+1$. By Definition $1, N_{\Gamma_{q}}\left((\varrho, 0, c)_{1}\right)=\left\{(c, 0, w)_{0}: w \in \mathbb{F}_{q}\right\} \cup\left\{(\varrho, \varrho, c)_{0}\right\}$; and $N_{\Gamma_{q}}\left((\varrho, \varrho, 0)_{1}\right)=\left\{(\varrho, 0, w)_{0}: w \in \mathbb{F}_{q}\right\} \cup\left\{(\varrho, \varrho, \varrho)_{0}\right\}$. Then $\left.(\varrho, \varrho, \varrho)_{1} \in N_{\Gamma_{q}}^{2}\left((\varrho, 0, c)_{1}\right)\right) \cap$ $\left.N_{\Gamma_{q}}^{2}\left((\varrho, \varrho, 0)_{1}\right)\right)$ for all $c \in \mathbb{F}_{q}$. Moreover, $N_{\Gamma_{q}}\left((c, 0, w)_{0}\right)=\left\{\left(a,-a c, a^{2} c+w\right)_{1}: a \in\right.$ $\left.\mathbb{F}_{q}\right\} \cup\left\{(\varrho, 0, c)_{1}\right\}$. Thus, for all $c_{1}, c_{2}, w_{1}, w_{2} \in \mathbb{F}_{q}, c_{1} \neq c_{2}$, we have $\left(a,-c_{1} a, a^{2} c_{1}+w_{1}\right)_{1}=$ $\left(a,-c_{2} a, a^{2} c_{2}+w_{2}\right)_{1}$ if and only if $a=0$ and $w_{1}=w_{2}$. Let $I_{A}=\bigcap_{a \in A} N_{\Gamma_{q}}^{2}(a)$. We conclude that $I_{A}=\left\{(\varrho, \varrho, \varrho)_{1}\right\} \cup\left\{(0,0, w)_{1}: w \in \mathbb{F}_{q}\right\}$ which implies that $\left|N_{\Gamma_{q}}[A]\right|+\left|I_{A}\right|=$ $(q+1)^{2}+2(q+1)$.

Since $N_{\Gamma_{q}}^{2}\left[(\varrho, \varrho, x)_{1}\right]=\bigcup_{j \in \mathbb{F}_{q}} N_{\Gamma_{q}}\left[(\varrho, x, j)_{0}\right] \cup N_{\Gamma_{q}}\left[(\varrho, \varrho, \varrho)_{0}\right]$ we obtain that $\left(N_{\Gamma_{q}}[A] \cup\right.$ $\left.I_{A}\right) \cap N_{\Gamma_{q}}^{2}\left[(\varrho, \varrho, x)_{1}\right]=\left\{(\varrho, \varrho, \varrho)_{0},(\varrho, \varrho, 0)_{1},(\varrho, \varrho, \varrho)_{1}\right\}$. Let $D=N_{\Gamma_{q}}[A] \cup I_{A} \cup N_{\Gamma_{q}}^{2}\left[(\varrho, \varrho, x)_{1}\right]$, then

$$
\begin{aligned}
|D| & =\left|N_{\Gamma_{q}}[A]\right|+\left|I_{A}\right|+\left|N_{\Gamma_{q}}^{2}\left[(\varrho, \varrho, x)_{1}\right]\right|-3 \\
& =(q+1)^{2}+2(q+1)+1+(q+1)+q(q+1)-3 \\
& =2 q^{2}+6 q+2 .
\end{aligned}
$$

Let us prove that $D$ is a perfect dominating set of $\Gamma_{q}$.

Let $H$ denote the subgraph of $\Gamma_{q}$ induced by $D$. Note that for $t, c \in \mathbb{F}_{q}$, the vertices $(x, t, c)_{1} \in N_{\Gamma_{q}}^{2}\left((\varrho, \varrho, x)_{1}\right)$ have degree 2 in $H$ because they are adjacent to the vertex $(\varrho, x, t)_{0} \in N_{\Gamma_{q}}(\varrho, \varrho, x)_{1}$ and also to the vertex $\left(-x^{-1} t, 0, x t+z\right)_{0} \in N_{\Gamma_{q}}(A)$. This implies that the vertices $(i, 0, j)_{0} \in N_{\Gamma_{q}}(A), i, j \in \mathbb{F}_{q}$, have degree 3 in $H$ and, also that the diameter of $H$ is 5 . Moreover, for $k \in \mathbb{F}_{q}$, the vertices $(\varrho, \varrho, k)_{0},(\varrho, 0, k)_{0} \in D$ have degree 2 in $H$ and the vertices $(\varrho, \varrho, j)_{1} \in D, j \in \mathbb{F}_{q} \backslash\{0, x\}$ have degree 1 in $H$. All other vertices in $D$ have degree $q+1$ in $H$.

Since the diameter of $H$ is 5 and the girth is $8,\left|N_{\Gamma_{q}}(v) \cap D\right| \leqslant 1$ for all $v \in V\left(\Gamma_{q}\right) \backslash D$, and also for all distinct $d, d^{\prime} \in D$ we have $\left(N_{\Gamma_{q}}(d) \cap N_{\Gamma_{q}}\left(d^{\prime}\right)\right) \cap\left(V\left(\Gamma_{q}\right) \backslash D\right)=\emptyset$. Then, $\left|N_{\Gamma_{q}}(D) \cap\left(V\left(\Gamma_{q}\right) \backslash D\right)\right|=q^{2}(q-2)+2 q(q-1)+(q-2) q+q^{2}(q-1)=2 q^{3}-4 q=\left|V\left(\Gamma_{q}\right) \backslash D\right|$. Hence $\left|N_{\Gamma_{q}}(v) \cap D\right|=1$ for all $v \in V\left(\Gamma_{q}\right) \backslash D$. Thus $D$ is a perfect dominating set of $\Gamma_{q}$. 
Definition 3. Let $q \geqslant 4$ be a prime power and let $x \in \mathbb{F}_{q} \backslash\{0,1\}$. Define $G_{q}^{x}$ as the $q$ regular graph of order $2 q\left(q^{2}-2\right)$ constructed by removing from $\Gamma_{q}$ its perfect dominating set $D$ given in Proposition 2.

Lemma 4. The q-regular graph $G_{q}^{x}$ in Definition 3 has girth exactly 8.

Proof. The graph $G_{q}^{x}$, by Definition 3 is $\Gamma_{q}$ minus a perfect dominating set $D$ so it clearly has girth at least 8 , and since it is bipartite its girth must be even. However, Moore's bound on the minimum number of vertices of a q-regular graph of girth 10 is $2\left(\sum_{i=0}^{4}(q-1)^{4}\right)$. Since the order of $G_{q}^{x}$ is $2 q\left(q^{2}-2\right)<2\left(\sum_{i=0}^{4}(q-1)^{4}\right)$, for all $q \geqslant 2$, $G_{q}^{x}$ must have girth exactly 8 .

Theorem 5. Let $q \geqslant 5$ be a prime power and let $G_{q}^{x}$ be the graph given in Definition 3 . Let $R=N_{G_{q}^{x}}\left(\left\{(\varrho, j, k)_{0}: j, k \in \mathbb{F}_{q}, j \neq 0,1, x\right\}\right) \cap N_{G_{q}^{x}}^{5}\left((\varrho, 1,0)_{0}\right)$. Then, the set

$$
S:=\bigcup_{j \in \mathbb{F}_{q}} N_{G_{q}^{x}}\left[(\varrho, 1, j)_{0}\right] \cup N_{G_{q}^{x}}[R]
$$

is a perfect dominating set in $G_{q}^{x}$ of cardinality $4 q^{2}-6 q$. Hence, $G_{q}^{x}-S$ is a $(q-1)$-regular graph of girth 8 and order $2 q(q-1)^{2}$.

Proof. Once $x \in \mathbb{F}_{q} \backslash\{0,1\}$ has been chosen to define $G_{q}^{x}$, to simplify notation, we will denote $G_{q}^{x}$ by $G_{q}$ throughout the proof. Denote by $P=\left\{(\varrho, j, k)_{0}: j, k \in \mathbb{F}_{q}, j \neq\right.$ $0,1, x\}$, then $R=N_{G_{q}}(P) \cap N_{G_{q}}^{5}\left((\varrho, 1,0)_{0}\right)$. Note that $d_{G_{q}}\left((\varrho, 1,0)_{0},(\varrho, j, k)_{0}\right)=4$, because according to Definition 1, $G_{q}$ contains the following paths of length four (see Figure 2): $(\varrho, 1,0)_{0}(1, b, 0)_{1}(w, w+b, w+2 b)_{0}(j, t, k)_{1}(\varrho, j, k)_{0}$, for all $b, j, t \in \mathbb{F}_{q}$ such that $b+w \neq 0$ due to the vertices $(j, 0, k)_{0}$ with second coordinate zero having been removed from $\Gamma_{q}$ to obtain $G_{q}$.

By Definition 1 we have $w+b=j w+t$ and $w+2 b=j^{2} w+2 j t+k$. If $w+b=0$, then $-w=b=t j^{-1}$ and $b=j t+k$ yielding that $t=\left(1-j^{2}\right)^{-1} j k$. This implies that $\left(j,\left(1-j^{2}\right)^{-1} j k, k\right)_{1} \in R$ is the unique neighbor in $R$ of $(\varrho, j, k)_{0} \in P$. Therefore every $(\varrho, j, k)_{0} \in P$ has a unique neighbor $(j, t, k)_{1} \in R$ leading to:

$$
|R|=|P|=q(q-3) .
$$

Thus, every $v \in N_{G_{q}}(R) \backslash P$ has at most $|R| / q=q-3$ neighbors in $R$ because for each $j$ the vertices from the set $\left\{(\varrho, j, k)_{0}: k \in \mathbb{F}_{q}\right\} \subset P$ are mutually at distance 6 (they were the $q$ neighbors in $\Gamma_{q}$ of the removed vertex $\left.(\varrho, \varrho, j)_{1}\right)$. Furthermore, every $v \in N_{G_{q}}(R) \backslash P$ has at most one neighbor in $N_{G_{q}}^{5}\left((\varrho, 1,0)_{0}\right) \backslash R$ because the vertices $\left\{(\varrho, 1, j)_{0}: j \in \mathbb{F}_{q}, j \neq 0\right\}$ are mutually at distance 6 . Therefore every $v \in N_{G_{q}}(R) \backslash P$ has at least two neighbors in $N_{G_{q}}^{3}\left((\varrho, 1,0)_{0}\right)$. Thus denoting $K=N_{G_{q}}\left(N_{G_{q}}(R) \backslash P\right) \cap N_{G_{q}}^{3}\left((\varrho, 1,0)_{0}\right)$ we have

$$
|K| \geqslant 2\left|N_{G_{q}}(R) \backslash P\right| \text {. }
$$

Moreover, observe that $\left(N_{G_{q}}(P) \backslash R\right) \cap K=\emptyset$ because these two sets are at distance four (see Figure 2). Since the elements of $P$ are mutually at distance at least 4 we obtain that $\left|N_{G_{q}}(P) \backslash R\right|=q|P|-|R|=(q-1)|P|$. Hence by $(2)$

$$
\left|N_{G_{q}}^{3}\left((\varrho, 1,0)_{0}\right)\right| \geqslant\left|N_{G_{q}}(P) \backslash R\right|+|K|=(q-1)|P|+|K|=(q-1) q(q-3)+|K| .
$$






Figure 2: Structure of the graph $G_{q}$. The perfect dominating set lies inside the dotted box.

Since $\left|N_{G_{q}}^{3}\left((\varrho, 1,0)_{0}\right)\right|=q(q-1)^{2}$ we obtain that $|K| \leqslant 2 q(q-1)$ yielding by (3) that $\left|N_{G_{q}}(R) \backslash P\right| \leqslant q(q-1)$. As $P$ contains at least $q$ elements mutually at distance $6, R$ contains at least $q$ elements mutually at distance 4 . Thus we have $\left|N_{G_{q}}(R) \backslash P\right| \geqslant q^{2}-q$. Therefore $\left|N_{G_{q}}(R) \backslash P\right|=q^{2}-q$ and all the above inequalities are actually equalities. Thus by (2) we get

$$
\left|N_{G_{q}}(R)\right|=q^{2}-q+|P|=2 q(q-2)
$$

and every $v \in N_{G_{q}}(R) \backslash P$ has exactly 1 neighbor in $N_{G_{q}}^{5}\left((\varrho, 1,0)_{0}\right) \backslash R$. Therefore we have

$$
\begin{aligned}
\left|N_{G_{q}}^{4}\left((\varrho, 1,0)_{0}\right) \backslash N_{G_{q}}(R)\right| & =\left|\bigcup_{j \in \mathbb{F}_{q} \backslash\{0\}}\left(N_{G_{q}}^{2}\left((\varrho, 1, j)_{0}\right) \cup P\right) \backslash N_{G_{q}}(R)\right| \\
& =q(q-1)^{2}+q(q-3)-2 q(q-2) \\
& =q(q-1)(q-2) .
\end{aligned}
$$

Let us denote by $E[A, B]$ the set of edges between any two sets of vertices $A$ and $B$. Then $\left|E\left[N_{G_{q}}^{3}\left((\varrho, 1,0)_{0}\right), N_{G_{q}}^{4}\left((\varrho, 1,0)_{0}\right)\right]\right|=q(q-1)^{3}$ and $\mid E\left[N_{G_{q}}^{3}\left((\varrho, 1,0)_{0}\right), N_{G_{q}}^{4}\left((\varrho, 1,0)_{0}\right) \backslash\right.$ $\left.N_{G_{q}}(R)\right] \mid=q(q-1)^{2}(q-2)$. Therefore,

$\left|E\left[N_{G_{q}}^{3}\left((\varrho, 1,0)_{0}\right), N_{G_{q}}(R)\right]\right|=q(q-1)^{3}-q(q-1)^{2}(q-2)=q(q-1)^{2}=\left|N_{G_{q}}^{3}\left((\varrho, 1,0)_{0}\right)\right|$,

which implies that every $v \in N_{G_{q}}^{3}\left((\varrho, 1,0)_{0}\right)$ has exactly one neighbor in $N_{G_{q}}(R)$. It follows that $S=\bigcup_{j \in \mathbb{F}_{q}} N_{G_{q}}\left[(\varrho, 1, j)_{0}\right] \cup N_{G_{q}}[R]$ is a perfect dominating set of $G_{q}$. Furthermore, by (2) and (4), $|S|=q^{2}+q+q(3 q-7)=4 q^{2}-6 q$. Therefore a $(q-1)$-regular graph of girth 8 can be obtained by deleting from $G_{q}$ the perfect dominating set $S$, see Figure 2 . This graph has order $2 q\left(q^{2}-2\right)-2 q(2 q-3)=2 q(q-1)^{2}$. 
Finally, as in the proof of Lemma 4 , recall that $G_{q}-S$ must have even girth since it is bipartite, and that the minimum number of vertices of a $(q-1)$-regular graph of girth 10 is $2\left(\sum_{i=0}^{4}(q-2)^{4}\right)$. The order of $G_{q}-S$ is $2 q(q-1)^{2}<2\left(\sum_{i=0}^{4}(q-2)^{4}\right)$, for all $q \geqslant 5$, a in the hypothesis. Therefore, $G_{q}-S$ has girth 8 .

\section{References}

[1] M. Abreu, M. Funk, D. Labbate, V. Napolitano. On (minimal) regular graphs of girth 6. Australas. J. Combin., 35:119-132, 2006.

[2] M. Abreu, M. Funk, D. Labbate, V. Napolitano. A family of regular graphs of girth 5. Discrete Math., 308(10):1810-1815, 2008.

[3] M. Abreu, G. Araujo-Pardo, C. Balbuena, D. Labbate. Families of small regular graphs of girth 5. Discrete Math., 312:2832-2842, 2012.

[4] G. Araujo, C. Balbuena, T. Héger. Finding small regular graphs of girths 6, 8 and 12 as subgraphs of cages. Discrete Math., 310(8):1301-1306, 2010.

[5] E. Bannai, T. Ito. On finite Moore graphs. J. Fac. Sci., Univ. Tokio, Sect. I A Math 20:191-208, 1973.

[6] C. Balbuena. Incidence matrices of projective planes and other bipartite graphs of few vertices. Siam J. Discrete Math., 22(4):1351-1363, 2008.

[7] C. Balbuena. A construction of small regular bipartite graphs of girth 8. Discrete Math. Theor. Comput. Sci., 11(2):33-46, 2009.

[8] L. M. Batten. Combinatorics of finite geometries. Cambridge University Press, Cambridge, UK, 1997.

[9] C.T. Benson. Minimal regular graphs of girth eight and twelve. Canad. J. Math., 18:1091-1094, 1966.

[10] L. Beukemann, K. Metsch. Regular Graphs Constructed from the Classical Generalized Quadrangle $Q(4, q)$. J. Combin. Designs, 19:70-83, 2010.

[11] J. A. Bondy, U. S. R. Murty. Graph Theory. Springer Series: Graduate Texts in Mathematics, Vol. 244, 2008.

[12] P. Erdős, H. Sachs. Reguläre Graphen gegebener Taillenweite mit minimaler Knotenzahl. Wiss. Z. Uni. Halle (Math. Nat.), 12: 251-257, 1963.

[13] G. Exoo. A Simple Method for Constructing Small Cubic Graphs of Girths 14, 15 and 16. Electron. J. Combin., 3(1):\#R30, 1996.

[14] G. Exoo, R. Jajcay. Dynamic cage survey. Electron. J. Combin., 15:\#DS16, 20082011-2013.

[15] A. Gács, T. Héger. On geometric constructions of $(k, g)$-graphs. Contrib. to Discrete Math., 3(1):63-80, 2008.

[16] C. Godsil, G. Royle. Algebraic Graph Theory. Springer, New York, 2000. 
[17] T. W. Haynes, S. T. Hedetniemi, P. J. Slater. Fundamentals of domination in graphs. Monogr. Textbooks Pure Appl. Math., 208, Dekker, New York, 1998.

[18] F. Lazebnik, V. A. Ustimenko, A. J. Woldar. Upper bounds on the order of cages. Electron. J. Combin., 4(2):\#R13, 1997.

[19] H. van Maldeghem. Generalized Polygons. Birkhauser, Basel 1998.

[20] M. Meringer. Fast generation of regular graphs and construction of cages. J. Graph Theory, 30:137-146, 1999.

[21] M. O'Keefe, P. K. Wong. The smallest graph of girth 6 and valency 7. J. Graph Theory, 5:79-85, 1981.

[22] S. E. Payne. Affine representation of generalized quadrangles. J. Algebra, 51:473-485, 1970.

[23] W. T. Tutte. A family of cubical graphs. Proc. Cambridge Philos. Soc., 43:459-474, 1947. 


\section{Corrigendum - Added May 18, 2021}

With respect to the original version of the paper, we actually construct a new infinite family of $(q-1)$-regular graphs of girth 8 and order $2 q\left(q^{2}-q-4\right)$ for all prime powers $q \geqslant 5$, which are the smallest known so far whenever $q-1$ is not a prime power or a prime power plus one itself.

The main difference is that we can no longer prove the existence of a perfect dominating set of the $q$-regular graphs in Definition 3 and Lemma 4 (the previous one was wrong for $q \geqslant 7$, it worked only for $q=5$ ). Therefore, we cannot construct the infinite family of $(q-1)$-regular graphs of girth 8 and order $2 q(q-1)^{2}$ for all prime powers $q$ as originally stated in Theorem 5 .

Instead, we use the following definition: if $G$ is $k$-regular, a subset $S \subset V(G)$ is $a$ quasi-perfect dominating set of $G$ if $G-S$ is $(k, k-1)$-regular and the set of vertices of degree $k$ in $G-S$ is either empty or it induces a perfect matching. We prove the existence of a quasi-perfect dominating set of the previously constructed $q$-regular graphs which allows us to construct a new infinite family of $(q-1)$-regular graphs of girth 8 and order $2 q\left(q^{2}-q-4\right)$ for all odd prime powers $q$ (cf. Theorem 5 ). These are the smallest $(q-1)$-regular graphs of girth 8 known so far for $q \geqslant 16$ whenever $q-1$ is not a prime power or a prime power plus one itself. Previously, the smallest known $(q-1,8)$-graphs, for $q$ a prime power, were those of order $2 q\left(q^{2}-q-1\right)$ which appeared in [7].

In this corrigendum we construct $(q-1)$-regular graphs of girth 8 with $2 q\left(q^{2}-q-4\right)$ vertices, for every prime power $q \geqslant 5$. To this purpose we use the coordinates for a Moore $(q+1,8)$-cage $\Gamma_{q}$ stated in Definition 1 .

In definition 3 we no longer need $x \in \mathbb{F}_{q} \backslash\{0,1\}$, but it is enough to have $x \in \mathbb{F}_{q} \backslash\{0\}$. We rewrite it for completion. The proof that it has girth 8 remains the same.

Definition 3. Let $q \geqslant 4$ be a prime power and let $x \in \mathbb{F}_{q} \backslash\{0\}$. Define $G_{q}^{x}$ as the $q$-regular graph of order $2 q\left(q^{2}-2\right)$ constructed by removing from $\Gamma_{q}$ its perfect dominating set $D$ given in Proposition 2.

Lemma 4. The q-regular graph $G_{q}^{x}$ in Definition 3 has girth exactly 8.

What follows are the new and corrected results:

Theorem 5. Let $q \geqslant 5$ be an odd prime power and let $G_{q}^{x}$ be the graph given in Definition 3 for some $x \in \mathbb{F}_{q} \backslash\{0,1,-1\}$. It is also required that if $q \equiv 1(\bmod 4)$ and $q>5$, then $x$ must satisfy that $x^{2}+1=0$. Let $R=\left\{(1, b,-2 b)_{1},(-1, b, 2 b)_{1}: b \in \mathbb{F}_{q}\right\}$, and let $y \in \mathbb{F}_{q} \backslash\{0,1,-1, x\}$; it is also required that if $q \equiv 1 \quad(\bmod 4)$ and $q>5$, then $y=x^{-1}$. Then, the set

$$
S:=\bigcup_{j \in \mathbb{F}_{q}} N_{G_{q}^{x}}\left[(\varrho, y, j)_{0}\right] \cup N_{G_{q}^{x}}[R]
$$


is a quasi-perfect dominating set in $G_{q}^{x}$ of cardinality $2 q^{2}+4 q$. The set of edges $M=$ $\left\{\left\{\left(a, b,-b\left(a+a^{-1}\right)\right)_{1},\left(\varrho, a,-b\left(a+a^{-1}\right)\right)_{0}\right\}: a, b \in \mathbb{F}_{q}, a \notin\{0,1,-1, x, y\}\right\}$ is empty if $q=5$ or a matching if $q \geqslant 7$ and $G_{q}^{x}-S-M$ is a $(q-1)$-regular graph of girth 8 and order $2 q\left(q^{2}-q-4\right)$.

Proof. To simplify notation, we will denote $G_{q}^{x}$ by $G$ throughout the proof. Observe that $N_{G}\left(\left\{(1, b,-2 b)_{1}: b \in \mathbb{F}_{q}\right\}=\left\{(j, t, j)_{0}: j, t \in \mathbb{F}_{q}, t \neq 0\right\} \cup\left\{(\varrho, 1, c)_{0}: c \in \mathbb{F}_{q}\right\}\right.$, because recall that the vertices $(j, 0, k)_{0}$ with second coordinate zero have been removed from $\Gamma_{q}$ to obtain $G$. Similarly, $N_{G}\left(\left\{(-1, b, 2 b)_{1}: b \in \mathbb{F}_{q}\right\}=\left\{(j, t, j)_{0}: j, t \in \mathbb{F}_{q}, t \neq 0\right\} \cup\right.$ $\left\{(\varrho,-1, c)_{0}: c \in \mathbb{F}_{q}\right\}$, yielding that $\left|N_{G}[R]\right|=2 q+q(q-1)+2 q=q^{2}+3 q$. Moreover, since $y \in \mathbb{F}_{q} \backslash\{0,1,-1, x\}$, it follows that the set $\bigcup_{j \in \mathbb{F}_{q}} N_{G}\left[(\varrho, y, j)_{0}\right]=\left\{(y, b, c)_{1}: b, c \in \mathbb{F}_{q}\right\}$ is disjoint with $N_{G}[R]$. Therefore $|S|=2 q^{2}+4 q$. Let us show that $S$ is a quasi-perfect dominating set. Observe that $(j, t, k)_{0} \in V(G), j, t, k \in \mathbb{F}_{q}, t \neq 0$, is adjacent to exactly one vertex $\left(y, t-y j, y^{2} j-2 y t+k\right)_{1}$ starting by $y$. This implies that, once removed from $G$ the set $\bigcup_{j \in \mathbb{F}_{q}} N_{G}\left[(\varrho, y, j)_{0}\right]$, all the vertices $(j, t, k)_{0}$ will have degree $q-1$ and the vertices $(\varrho, a, c)_{0}, a \in \mathbb{F}_{q} \backslash\{0, x, y\}, c \in \mathbb{F}_{q}$ will have degree $q$. Thus, once the points $(j, t, j)_{0}$, $j, t \in \mathbb{F}_{q}, t \neq 0$, have been removed from $G$, only the vertices $(a, b, c)_{1}$ adjacent to vertices $(j, 0, j)_{0}$ in $\Gamma_{q}$ will remain with degree $q$ in $G-S$. Therefore, the vertices of the set $M=\left\{\left(a, b,-b\left(a+a^{-1}\right)\right)_{1},(\varrho, a, c)_{0}: a, b, c \in \mathbb{F}_{q}, a \notin\{0,1,-1, x, y\}\right\}$ have degree $q$ in $G-S$. Suppose $q \equiv 3 \quad(\bmod 4)$, we know that -1 has no square root in $\mathbb{F}_{q}$. This implies that $a+a^{-1} \neq 0$ for all $a \in \mathbb{F}_{q} \backslash\{0,1,-1, x, y\}$ and for every $b \in \mathbb{F}_{q}$ there is a unique value $-b\left(a+a^{-1}\right)$ associated to $a$. Since $\left(a, b,-b\left(a+a^{-1}\right)\right)_{1}$ is adjacent to $\left(\varrho, a,-b\left(a+a^{-1}\right)_{0}\right.$, it follows that the subgraph induced by $M$ is a matching. Finally, suppose that $q \equiv 1$ $(\bmod 4)$, we know that -1 has two square roots in $\mathbb{F}_{q}$, which are $x$ and $x^{-1}$ because by hypothesis $x^{2}+1=0$. Hence $a+a^{-1} \neq 0$ for all $a \in \mathbb{F}_{q} \backslash\{0,1,-1, x, y\}$ and we proceed as before. We conclude that $S$ is a quasi-perfect dominating set.

Theorem 6. Let $q \geqslant 8$ be an even prime power and $x \in \mathbb{F}_{q} \backslash\{0,1\}$. Let $G_{q}^{x}$ be the graph given in Definition 3. Let $m \in \mathbb{F}_{q} \backslash\{0,1, x\}$ and let $R=\left\{\left(m, b,\left(m+m^{-1}\right) b\right)_{1},\left(m^{-1}, b,(m+\right.\right.$ $\left.\left.\left.m^{-1}\right) b\right)_{1}: b \in \mathbb{F}_{q}\right\}$. Then, the set

$$
S:=\left\{N_{G_{q}^{x}}\left[(\varrho, 1, c)_{0}\right]: c \in \mathbb{F}_{q}\right\} \cup N_{G_{q}^{x}}[R]
$$

is a quasi-perfect dominating set in $G_{q}^{x}$ of cardinality $2 q^{2}-4 q$. The set of edges $M=$ $\left\{\left\{\left(\varrho, a,\left(a+a^{-1}\right) b\right)_{0},\left(a, b,\left(a+a^{-1}\right) b\right)_{1}\right\}: a \in \mathbb{F}_{q}, a \neq 0,1, x, m, m^{-1}\right\}$ satisfies for $q \geqslant 8$ that $G_{q}^{x}-S-M$ is a $(q-1)$-regular graph of girth 8 and order $2 q\left(q^{2}-q-4\right)$.

Proof. Once $x \in \mathbb{F}_{q} \backslash\{0,1\}$ has been chosen to define $G_{q}^{x}$, to simplify notation, we will denote $G_{q}^{x}$ by $G$ throughout the proof. We have $d_{G}\left((\varrho, a, c)_{0},\left(\varrho, a, c^{\prime}\right)_{0}\right)=6$, for all $a \in \mathbb{F}_{q}, a \neq 0, x$, and two distinct $c, c^{\prime} \in \mathbb{F}_{q}$ (these two vertices were neighbors in $\Gamma_{q}$ of vertex $(\varrho, \varrho, a)_{1}$ eliminated from $\Gamma_{q}$ to obtain $\left.G\right)$. Hence, after the deletion of $A=$ $\left\{N_{G_{q}^{x}}\left[(\varrho, 1, c)_{0}\right]: c \in \mathbb{F}_{q}\right\}$ the resulting graph $H$ has degrees $q-1$ and $q$ and every vertex of the form $(i, j, k)_{0}$ with $i \in \mathbb{F}_{q}$ has degree $q-1$. Indeed, it is enough to note that $(i, j, k)_{0},(1, i+j, i+k)_{1},(\varrho, 1, i+k)_{0}$ is a path of length two. Also observe that $|A|=q(q+1)$. 
Let $m \in \mathbb{F}_{q} \backslash\{0,1, x\}$, then $m \neq m^{-1}$. Consider the sets: $W_{1}=\left\{\left(m, b,\left(m+m^{-1}\right) b\right)_{1}\right.$ : $\left.b \in \mathbb{F}_{q}\right\}$ and $W_{2}=\left\{\left(m^{-1}, b,\left(m+m^{-1}\right) b\right)_{1}: b \in \mathbb{F}_{q}\right\}$. Let us show that $N_{G}\left(W_{1}\right) \backslash\left\{(\varrho, m, i)_{0}:\right.$ $\left.i \in \mathbb{F}_{q}\right\}=N_{G}\left(W_{2}\right) \backslash\left\{\left(\varrho, m^{-1}, k\right)_{0}: k \in \mathbb{F}_{q}\right\}$. It is enough to show that if $\ell_{1} \in W_{1}$ and $\ell_{2} \in W_{2}$ we have $d_{G}\left(\ell_{1}, \ell_{2}\right)=2$. Indeed, $\ell_{1}=\left(m, b,\left(m+m^{-1}\right) b\right)_{1},\left(w, m w+b, m^{2} w+(m+\right.$ $\left.\left.m^{-1}\right) b\right)_{0},\left(m^{-1}, c,\left(m+m^{-1}\right) c\right)_{1}=\ell_{2}$ is a path of length two, with $c=\left(m+m^{-1}\right) w+b$. Therefore, $N_{G}\left(W_{1}\right) \backslash\left\{(\varrho, m, i)_{0}: i \in \mathbb{F}_{q}\right\}=N_{G_{q}}\left(W_{2}\right) \backslash\left\{\left(\varrho, m^{-1}, k\right)_{0}: k \in \mathbb{F}_{q}\right\}$ as claimed. Then, the cardinality of $N_{G}[R]$ is $\left|N_{G}\left[W_{1}\right]\right|+\left|W_{2}\right|+\left|\left\{\left(\varrho, m^{-1}, k\right)_{0}: k \in \mathbb{F}_{q}\right\}\right|=q^{2}+3 q$.

Let $v \notin N_{G}[R]$ be such that $v=(r, s, t)_{1}$ with $r \in \mathbb{F}_{q}, r \neq 1$, and suppose that $\left|N_{G}(v) \cap N_{G}[R]\right| \geqslant 1$. If $r=m$ and $\left(m, b,\left(m+m^{-1}\right) b\right)_{1},\left(\varrho, m,\left(m+m^{-1}\right) b\right)_{0},(m, s, t)_{1}$ is a path of length 2 , then $t=\left(m+m^{-1}\right) b$. Analogously, if $r=m^{-1}$, then $t=$ $\left(m+m^{-1}\right) b$. If $t \neq\left(m+m^{-1}\right) b$, then $d_{G}\left(\left(m, b,\left(m+m^{-1}\right) b\right)_{1},(m, s, t)_{1}\right)=4$ because $d_{G}\left(\left(\varrho, m,\left(m+m^{-1}\right) b\right)_{0},(\varrho, m, t)_{0}\right)=6$ and $\left(m, b,\left(m+m^{-1}\right) b\right)_{1} \sim\left(\varrho, m,\left(m+m^{-1}\right) b\right)_{0}$, $(m, s, t)_{1} \sim(\varrho, m, t)_{0}$. Hence, any path of length two $\left(m, b,\left(m+m^{-1}\right) b\right)_{1},(w, w m+$ $\left.b, w m^{2}+\left(m+m^{-1}\right) b\right)_{0},(r, s, t)_{1}$ satisfies that $r \neq m, w \neq m^{-1} b$ because the elements $(i, 0, k)_{0}$ are not in $G$ and,

$m w+b=r w+s$, or equivalently $w(m+r)=b+s$ and

$m^{2} w+\left(m+m^{-1}\right) b=r^{2} w+t$, or equivalently $w\left(m^{2}+r^{2}\right)=\left(m+m^{-1}\right) b+t$.

Since $q$ is even, $\left(m^{2}+r^{2}\right)=(m+r)^{2}$, and it follows that $(m+r)(b+s)=\left(m+m^{-1}\right) b+t$, which implies that $t=\left(m^{-1}+r\right) b+(m+r) s$.

Analogously, if $\left(m^{-1}, b,\left(m+m^{-1}\right) b\right)_{1},\left(w, w m^{-1}+b, w m^{-2}+\left(m+m^{-1}\right) b\right)_{0},(r, s, t)_{1}$ is a path of length 2 , then $r \neq m^{-1}$ and $t=(m+r) b+\left(m^{-1}+r\right) s$.

Suppose by contradiction that $\left|N_{G}(v) \cap N_{G}[R]\right| \geqslant 2$, which implies that there is a path $\ell_{1}, p_{1}, v, p_{2}, \ell_{2}$ of length four with $p_{i} \in N(R)$ and $\ell_{i} \in R, i=1,2$. This means that $\ell_{1}, \ell_{2} \in W_{1}$ or $\ell_{1}, \ell_{2} \in W_{2}$, because $d_{G}\left(\ell_{1}, \ell_{2}\right)=4$. Suppose that both $\ell_{1}, \ell_{2} \in W_{1}$, (the case $\ell_{1}, \ell_{2} \in W_{2}$ is analogous) then $\ell_{i}=\left(m, b_{i},\left(m+m^{-1}\right) b_{i}\right)_{1}, i=1,2$, with $b_{1} \neq b_{2}$ because we are assuming that $\ell_{1} \neq \ell_{2}$. Then $v=(r, s, t)$ with either $t=\left(m+m^{-1}\right) b_{1}=\left(m+m^{-1}\right) b_{2}$ or with $t=\left(m^{-1}+r\right) b_{1}+(m+r) s=\left(m^{-1}+r\right) b_{2}+(m+r) s$. In the former case $b_{1}=b_{2}$ which is a contradiction. In the later case, $\left(m^{-1}+r\right)\left(b_{1}+b_{2}\right)=0$. Since $b_{1} \neq b_{2}$ it follows that $r=m^{-1}$ which is a contradiction. Then $\left|N_{G}(v) \cap N_{G}[R]\right|=1$.

Let $H^{\prime}=G-\left(\left\{N_{G_{q}^{x}}\left[(\varrho, 1, c)_{0}\right]: c \in \mathbb{F}_{q}\right\} \cup N_{G_{q}^{x}}[R]\right)$. The degree of $(\varrho, a, c)_{0}$ with $a, c \in \mathbb{F}_{q}$, $a \neq 0,1, x, m, m^{-1}$, is $q$ in $H^{\prime}$. And every element $(a, b, c)_{1}$ such that $d_{G}\left(\ell,(a, b, c)_{1}\right)=4$ for some $\ell \in R$ and $d_{G}\left((\varrho, 1, c)_{0},(a, b, c)_{1}\right)=5$ for some $c \in \mathbb{F}_{q}$ has also degree $q$ in $H^{\prime}$.

For all $b, w \in \mathbb{F}_{q}$ we have the following shortest paths:

$(\varrho, 1,0)_{0},(1, b, 0)_{1}(w, w+b, w)_{0},\left(a, w+b+a w, a^{2} w+w\right)_{1},\left(\varrho, a, a^{2} w+w\right)_{0},\left(a, a w, a w\left(a+a^{-1}\right)\right)_{1}$

$$
\left(m, b,\left(m+m^{-1}\right) b\right)_{1},\left(0, b,\left(m+m^{-1}\right) b\right)_{0},(\varrho, b, 0)_{1},\left(0, b,\left(a+a^{-1}\right) b\right)_{0},\left(a, b,\left(a+a^{-1}\right) b\right)_{1}
$$

Therefore the elements $\left(a, b,\left(a+a^{-1}\right) b\right)_{1}$ with $a \neq 0,1, x, m, m^{-1}$ have degree $q$ in $H^{\prime}$. Since $\left(a, b,\left(a+a^{-1}\right) b\right)_{1}$ is adjacent to $(\varrho, a, c)_{0}$ by deleting edges $M=\left\{\left\{\left(\varrho, a, a+a^{-1}\right) b\right)_{0},(a, b,(a+\right.$ $\left.\left.\left.\left.a^{-1}\right) b\right)_{1}\right\}: a \in \mathbb{F}_{q}, a \neq 0,1, x, m, m^{-1}\right\}$ from $H^{\prime}$ we obtain a $(q-1)$-regular graph of order $2 q\left(q^{2}-q-4\right)$ and the result holds. 